Edward P O’Brien, Catherin M Hill

Assessing the potential of non-harmful, natural feeding deterrents tested on captive primates, International Journal of Pest Management, (forthcoming)

DOI:

This version is available: https://radar.brookes.ac.uk/radar/items/Ofe4ed03-3534-4cdd-9789-57f4fc507183/1/

Available on RADAR: 02.03.2017

Copyright $(\mathbb{C}$ and Moral Rights are retained by the author(s) and/ or other copyright owners. A copy can be downloaded for personal non-commercial research or study, without prior permission or charge. This item cannot be reproduced or quoted extensively from without first obtaining permission in writing from the copyright holder(s). The content must not be changed in any way or sold commercially in any format or medium without the formal permission of the copyright holders.

This document is the authors' final accepted manuscript. 


\section{Assessing the potential of non-harmful, natural feeding deterrents tested on captive primates}

\section{Edward P. O'Brien ${ }^{1 *}$ and Catherine M. Hill ${ }^{2}$}

\section{Author affiliations:}

${ }^{1}$ Edward P. O’Brien

${ }^{2}$ Department of Social Sciences, Faculty of Humanities \& Social Sciences, Oxford Brookes University, Gipsy Lane, Oxford OX3 0BP, UK.

* Corresponding author:

Catherine M. Hill

Department of Social Sciences, Faculty of Humanities \& Social Sciences, Oxford Brookes University, Gipsy Lane, Oxford OX3 0BP

Telephone: $+44(0) 1865483757$

Fax: $+44(0) 1865483937$

Email: cmhill@brooks.ac.uk 


\begin{abstract}
Crop damage by non-human primates, can cause friction between local people, government wildlife agencies and conservationists. Consequently, developing effective, non-lethal methods to protect crops against primate foraging could benefit farmers, reduce conflicts between interest groups, and even promote primate conservation The purpose of this research was to (i) develop a simple, economical protocol for testing the deterrent properties of nonlethal plant substances on captive primates prior to testing in the field; (ii) examine the preliminary effectiveness of neem (Azadirachta indica) and ocimum (Ocimum kilimandscharicum) essential oils, and spent coffee grounds (SCG) as feeding deterrents in captive macaques. The test methods developed involved exposing primates to possible feeding deterrents whilst feeding and comparing to a control. This was used to identify likely repellent substances, which could then be tested in the field to determine their effectiveness against crop damage. SCG were most effective at deterring the macaques; ocimum and neem essential oils were less effective though animals appeared less willing to access food when protected by these oils than they were under control conditions.
\end{abstract}

Keywords: Captive, Crop-raiding, Deterrent, Essential-oils, Human-wildlife conflict, Macaque 


\section{Introduction}

Primates forage on crops for a variety of different reasons including habitat change, as part of an optimal foraging strategy or even because of apparent food preferences (Strum,2010; Riley et. al., 2013; MacLarnon et al. 2015; Seiler and Robbins, 2015). Certain primate species, such as baboons and macaques, are prolific crop feeders across Africa and Asia, and the consequences of their crop foraging activities are experienced by local people, whose livelihoods can be significantly impacted (e.g. Sajet al. 2001; Webber et al.2007; Priston and McLennan 2013; Humle and Hill, 2016). A common response by those affected is to hunt the animals (Woodroffe et al, 2005), thus further threatening the long-term survival of certain species, including Arunachal macaques (Macaca munzala) and chimpanzees (Pan troglodytes) (Kumar et al. 2008; McLennan and Hill 2012).

A variety of different methods to reduce crop losses from primate foraging activity have been proposed in the literature but very few have ever been tested systematically (Hill and Wallace, 2012). Guarding is a strategy that is adopted, to varying degrees, by many farmers throughout primate range countries. It can be very effective if farmers engage in active guarding, i.e., they regularly patrol field boundaries and actively chase primates out of fields (Hill and Wallace 2012). However, this requires a significant time investment on the part of the farmer (Wang et al. 2006), and thus incurs significant lost opportunity costs (Hill and Wallace, 2012; Mackenzie et al 2015). Alternative deterrents, although not always as effective, require less labour and investment of time than guarding, and therefore may be preferred by farmers. 
Hill and Wallace (2012) systematically tested a range of non-lethal crop foraging deterrents in association with local stakeholders in rural Uganda, identifying camphor basil (Ocimum kilimandscharicum), or ocimum. We use the terms 'deterrent' as defined by Hill and Wallace (2012), i.e., a deterrent is "any technique intended to protect crops from damage by animals at any stage irrespective of how or at what stage of a crop raiding [crop foraging] event the technique influences raider behaviour." (Hill and Wallace, 2012; p. 2570). An innate aversion to unpleasant or "fishy" odours has been identified in capuchin monkeys (Cebus paella) (Ueno 1994), and a number of plant secondary metabolites have shown promise for repelling rodents (Harris et al. 2016). However, the testing of new deterrents in situ is likely to result in significant costs for both the researcher and farmer, particularly where farmers are encouraged to adopt untested methods that prove less effective than anticipated, rendering farmers vulnerable to increased crop losses alongside the time or cash costs of implementing said methods. The aims of this project are: (i) to pilot a research protocol for trialling nonlethal plant derivatives on captive primates as a precursor to developing in situ trials to reduce crop losses to wild primates; and (ii) to test the effectiveness of neem (Azadirachta indica) and ocimum (Ocimum kilimandscharicum) essential oils, and spent coffee grounds (SCG), as feeding deterrents in captive macaques.

Camphor basil (Ocimum kilimandscharicum) was chosen because of its aromatic properties, which have previously been postulated to have an olfactory deterrent effect on primates when incorporated into barriers around field boundaries (Hill and Wallace 2012). Neem (Azadirachta indica), also an olfactory deterrent, was chosen because of its strong, garlic-like odour and the fact that it can deter rats from feeding (Goah 1999). A third substance, spent coffee grounds (SCG), was chosen because primates show an aversion to caffeine (Laska et al. 2009), so it is anticipated that SCG would provide a combined taste and olfactory 
deterrent. SCG is an abundant resource with over six million tonnes produced as waste each year (Mussatto et al. 2011).

\section{Methods}

The three test substances were trialled on a group of Sulawesi crested macaques (Macaca nigra) at Newquay Zoo, Cornwall, between $4^{\text {th }}$ and $10^{\text {th }}$ June 2013. The animals were housed in an indoor enclosure $\left(8 \mathrm{~m}^{2}\right)$ furnished with ropes, tyres and wooden structures. They also had access to a grass outdoor area $\left(15 \mathrm{~m}^{2}\right)$ with large wooden climbing structures and ropes (see Figure 1). The macaques were fed a daily diet of mainly root vegetables at 10am and $4 \mathrm{pm}$, with additional leafy greens at $12: 30 \mathrm{pm}$. The study group comprised five adults: an adult male, the dominant female, and three subordinate females. The average age of the study individuals was 12 years old, and all were captive bred.

\section{Experimental Design}

The most common method for testing taste preferences is the two-bottle preference test (Richter and Campbell 1940). This test involves offering an animal the choice of two feeding sites, a control and experimental bottle, and recording the feeding time spent at each. Our experimental protocol is derived from this approach, but in order to mimic primate crop foraging scenarios more closely, primates had access to natural foods rather than a sugar solution while being exposed to the test substances. Group feeding was measured by feeding frequency and duration, unlike traditional methods which have examined primates individually; this is a more realistic way of observing primate feeding. A macaque species was chosen for this research because all members of the genus Macaca are known to feed on crops (Priston \& McLennan, 2013). 


\section{Experimental Protocol}

The first phase, the habituation phase, was to condition the animals to associate the test feeding stations with eating. Two large mesh doors $2 \mathrm{~m}$ apart were used with two buckets attached to each (see elevation in Figure 1), which were spaced $60 \mathrm{~cm}$ apart, as this spacing was sufficient to prevent odour drift. Specially modified plastic buckets were used to create the feeding stations because they are cheap, readily available and easy to modify, thereby allowing the experiments to be easily replicated. Additionally, plastic buckets are regularly used for enrichment by zoos, including Newquay, and therefore are unlikely to cause the animals unnecessary stress. Moreover, the same plastic buckets could be modified into two slightly different feeding stations to test the essential oils and spent coffee grounds (SCG) (see Figures 2 and 3). Feeding stations were attached to the outside of the enclosures, facilitating the attachment and removal of the apparatus between trials without needing to enter the enclosures. Positioning them thus also ensured that it was easy for the researcher to observe which primates were feeding and for how long.

Four buckets were used in all habituation and experimental trials. Fifty grammes of split peas were mixed with $80 \mathrm{~g}$ of straw in each feeding station to increase foraging time. Split peas were chosen after discussion with the primate keeper because they are highly attractive to macaques and are small, requiring careful manipulation. The conditioning phase involved the provision of food in the feeding stations twice daily, over 3 consecutive days. Each conditioning trial was carried out 1 hour prior to the animals' scheduled feeding time, to ensure animals were hungry, motivated to forage for the split peas, and unlikely to become satiated during trials. Previous research suggests that five separate encounters is the number of exposures required for habituation to an unfamiliar food or smell to occur (Visalberghi et al.1998; Visalberghi et al. 2003; Laska et al. 2009). Consequently, five trials of each test 
substance were undertaken during the second, experimental phase, which immediately followed the habituation phase. At 9am each day during the experimental phase, the essential oils were tested. In two of the four feeding stations an essential oil was infused into hollofill fibre and placed under a false floor to allow the smell to permeate through. The remaining two feeding stations acted as a control and were identical apart from their lack of essential oils. Similarly, at $3 \mathrm{pm}$ on each of the 5 experimental days, spent coffee grounds (SCG) were tested against a sterile compost control. The test feeding stations contained $200 \mathrm{~g}$ of SCG, while the controls contained $200 \mathrm{~g}$ of sterile compost with the same moisture content, and $50 \mathrm{~g}$ of split peas mixed with each substrate along with the straw, as previously. Throughout both sets of trials the positions of the feeding stations were rotated to minimise potential bias due to feeding station position. Ocimum and neem were tested separately against the control condition throughout the trials.

\section{Data Collection}

A continuous sampling technique (Altmann 1974) was used to record the frequency and duration of feeding over a one hour observation period. Each observation period began as soon as the feeding stations were attached. Feeding was defined as foraging inside the feeding station or consuming items retrieved from the feeding station within $50 \mathrm{~cm}$ of the feeding station. Feeding frequency was defined as the amount of time spent foraging in the buckets; the animals may not have consumed everything foraged but were in contact with the foodstuffs. The amount of time each animal spent foraging was recorded with a stopwatch and measured in seconds. All data were collected by the first author.

\section{Data Analysis}


The assumptions of parametric statistics were not met due to the small sample size, which did not conform to a normal distribution; therefore, non-parametric tests were performed. For the essential oil trials, because the samples were not independent of one another, a Friedman's test was used to establish whether the differences in feeding duration and frequency were significant. ATA Wilcoxon signed ranks test was then used to determine whether there were significant differences in animal responses to pairs of substances, with a $p$ value of $<0.05$ being considered significant. For the SCG trials the comparison was between just two results, consequently the Wilcoxon signed ranks test was used. All analyses were carried out using SPSS for windows, version 19.0.

\section{Ethical Considerations}

The animals' welfare was of the highest concern during the development of these experiments. Using multiple feeding stations helped uphold welfare by minimizing the number of aggressive encounters associated with clumped food resources. This study conforms to the 'Guidelines for the treatment of animals in behavioural research and

teaching' (Society for the Study of Animal Behaviour 2006). The animals were closely observed for adverse reactions throughout the research and no adverse behaviour/reactions were noted. Research clearance was provided by Newquay Zoo after the completion of their ethics form.

\section{Results}

\section{Essential Oils}

There were noticeable differences between the times spent feeding at the test feeding stations, compared with the control conditions (Figure 4).The total time spent feeding at both the 
ocimum and neem test stations was approximately $84 \%$ less than time spent feeding at the control stations. The differences in time spent feeding between test and control stations is significant (Friedman's Test, $\mathrm{n}=5, \mathrm{p}=0.015$ ). A comparison between samples illustrates that differences exist between the time spent feeding at each of the essential oils feeding sites and the control sites, but not between time spent feeding at the ocimum and neem test sites, i.e., both essential oils appear to affect feeding behaviour to the same degree (Wilcoxon signed ranks test, $\mathrm{n}=3$, ocimum - control $\mathrm{p}=0.039$; neem - control $\mathrm{p}=0.042$; ocimum - neem $\mathrm{p}=0.498$ ). Additionally, no sign of habituation to the essential oils was recorded over the test period (Figure 5). Total feeding levels remained roughly the same or increasing in both groups (Figures 5 and 8 ).

However, notable differences were observed between the feeding frequency at the neem feeding stations and controls, with the neem feeding stations being frequented $50 \%$ less often, whilst the differences between the control and ocimum feeding stations were slightly lower at 35\% (Figure 6). The differences in feeding frequency were found to be significant (Friedman's Test, $\mathrm{n}=5, \mathrm{p}=0.009$ ), and a comparison between the samples illustrates that the differences lie between the essential oils and the control and not between ocimum and neem (Wilcoxon signed ranks test, $\mathrm{n}=3$, ocimum - control $\mathrm{p}=0.041$; neem - control $\mathrm{p}=0.034$; ocimum - neem $\mathrm{p}=0.102$ ).

\section{Spent Coffee Grounds}

Time spent feeding at the spent coffee grounds (SCG) feeding station was $90 \%$ less than at the control feeding station (Figure 7), and this difference in feeding duration was significant (Wilcoxon signed ranks test, $\mathrm{n}=5, \mathrm{p}=0.042$ ). There was a slight increase in time spent feeding 
at the control station on days 4 and 5, there were no distinct changes in the total levels of feeding duration over the evaluation period (Figure 8). Additionally, the control feeding stations were visited more than twice as often as the SCG test feeding stations (Figure 9), and this difference was statistically significant (Wilcoxon signed ranks test, $n=5, p=0.041$ ).

\section{Discussion}

\section{Initial Promise of Results}

Neem and ocimum essential oils both appear promising candidates in preliminary evaluation of deterrents. It is possible that the deterrent effect observed was as a consequence of the essential oils being unfamiliar odours to the monkeys (Ueno 1990); consequently it is recommended that further trials of both essential oils be undertaken to determine the length of the deterrent effect. This will inform how best to manage their use.

The results for the spent coffee grounds (SCG) trial were also encouraging. It is likely that the aversive smell or high caffeine content was responsible for the deterrent effect exhibited by the SCG. Further trials of SCG as a feeding deterrent should be undertaken, extending the period of exposure to determine whether the animals become habituated to SCG. If these provide similar results, then a pilot field study should be undertaken to determine application methods. This pilot study could utilise SCGs as a crop mulch or soil improver where, from the results of this study, it would be likely to act as a deterrent. Ocimum and Neem could be trialled as live scented hedges or as a paste applied to rope fences (e.g. Hill and Wallace 2012).

\section{Evaluating the Methods}


The research protocol described provides a quick, simple and economic method of checking the initial response of primates to feeding deterrents. This could therefore reduce the need for costly field trials. There are a number of advantages to this approach. It is cheap and easy to implement, and uses readily available materials, which means that it could be replicated anywhere in the world where there are suitable groups of captive primates, zoos willing to participate and available deterrent ingredients. Such trials enable costly field trips to then focus on substances which have already proven effective in pre-trials.

Using multiple feeding stations ensured that the food was distributed widely enough to prevent any increase in aggressive interactions between test animals associated with clumped food sources (Bocciaand Hijazi 1988). While this was not empirically recorded, no increase in aggression was noted by the observers. Additionally, this facilitated the testing of a group of animals, thereby allowing significant amounts of data to be collected within a restricted time period. Furthermore, because the experiment could be set up entirely from outside the enclosure, this minimised the potential stress experienced by the animals, as well as being time-efficient for the researchers when initiating each trial session.

Using a high-value foodstuff provides the test animals with a strong incentive to forage at the feeding stations consistently, and to at least attempt to forage at the deterrent stations. We argue that this replicates the high-energy foods which are frequently consumed by primates on farms (e.g. Naughton Treves 1998; Hill 2000; Riley 2007). Observed behaviour confirmed the value of using pea sized foods, because it was necessary to have an extended feeding time to gain enough data for a meaningful study. 
The mesh size of an enclosure is also critical because if the mesh is too large then subjects can withdraw a closed fist through it and there is a risk that they may simply retrieve large handfuls of the food-straw mixture and sort through it on the floor of their enclosure, thus reducing exposure to the deterrent. During the trials, regular attempts were made by the primates to unfasten the buckets and empty the contents onto the floor; therefore, it should be ensured that the mesh size will not allow the primates' full arm through it.

This method of testing possible deterrents has provided measurable results, which offers clear benefit for anyone planning field trials; it offers the opportunity for simple testing of natural feeding deterrents and informs new strategies.

\section{References}

Altmann J (1974) Observational study of behaviour: sampling methods. Behav 49: 3-4

Boccia M \& Hijazi A (1998) A foraging task reduces agonistic and stereotypic behaviors in pigtail macaque social groups. Lab Primate News 137: 1-4

Gaoh A (1999) Potential of Selected Natural Products as Repellents Against Vertebrate Pests of Crops. McGill University,Canada

Hansen C, Stolter C, Jacob J (2016) Plant Secondary Metabolites as Rodent Repellents. A Systematic Review. J Chem Ecology 42(9):970-983

Hill C (2000) Conflict of interest between people and baboons: crop raiding in Uganda. Int J of Primatology 21: 299-315

Hill C, Wallace G (2012) Crop protection and conflict mitigation: reducing the costs of living alongside non-human primates. Biodivers and Conserv 21: 2569-2587 
Kumar R, Gama N, Raghunath R, Sinha A, Mishra C (2008) In search of the munzala: distribution and conservation status of the newly-discovered Arunachal macaque (Macaca munzala). Oryx 42(3): 360-366

Laska M, Rivas Bautista R, Hernandez Salazar L (2009) Gustatory responsiveness to six bitter tastants in three species of nonhuman primates. J Chem Ecol 35: 560-571 Lee P, Priston N (2005) Human attitudes to primates: perceptions of pests, conflict and consequences for conservation. In: Paterson JD (eds) Commensalism and conflict: the primate-human interface, 1st ed Winnipeg, Manitoba, Hignell Printing pp 1-23 Mackenzie C, Sengupta R, Kaoser, R (2015) Chasing baboons or attending class: protected areas and childhood education in Uganda. Environmental Conservation $: 1-11$

McLennan M, Hill C (2012) Troublesome neighbours: Changing attitudes towards chimpanzees (Pan troglodytes) in a human-dominated landscape in Uganda. J Nat Conserv 20(4): 219-227

Mussatto S, Machado E, Martins S, Teixeira J (2011) Production, composition, and application of coffee and its industrial residues. Food and Bioprocess Tech 4: 661-672 Naughton-Treves L (1998) Predicting patterns of crop damage by wildlife around Kibale National Park, Uganda. Conserv Biol 12(1):156-168

Osborn F, Parker G (2002) Community-based methods to reduce crop loss to elephants: experiments in the communal lands of Zimbabwe. Pachyderm 33:32-38

Priston N, McLennan M (2013) Managing Humans, Managing Macaques: Human-Macaque Conflict in Asia and Africa. In The Macaque Connection, Springer, NewYork pp 225-250 Richter C, Campbell K (1940) Alcohol taste thresholds and concentrations of solution preferred by rats. Sci 91:507-508 
Riley E(2007) The human-macaque interface: conservation implications of current and future overlap and conflict in Lore Lindu National park, Sulawesi, Indonesia. Am Anthropol 109:473-484

Saj T, Sicotte P, Paterson J (2001) The conflict between vervet monkeys and farmers at the forest edge in Entebbe, Uganda. Afr J Ecol 39(2): 195-199

Sillero-Zubiri C, Switzer D (2001) Crop Raiding Primates: Searching for Alternative, Humane Ways to Resolve Conflict with Farmers in Africa. Wildlife Conservation Research Unit, Oxford University, Oxford

Society for the Study of Animals Behaviour (2006) Guidelines for the treatment of animals in behavioural research and teaching. Anim Behav 71:245-253

Strum S (1994) Prospects for management of primate pests. Rev Ecol (Terre Vie) 49:295306

Strum S (2010) The development of primate raiding: implications for management and conservation. Int J Primatology 31: 133-156

Ueno Y (1994) Olfactory discrimination of eight food flavors in the capuchin monkey, Cebus apella: comparison between fruity and fishy odors. Primates 35: 301-310

Visalberghi E, Valente M, Fragaszy D (1998) Social context and consumption of unfamiliarfoods by capuchin monkeys (Cebus apella) over repeated encounters. Am J Primatology 45(4): 367-380

Visalberghi E, Sabbatini G, Stammati M, Addessi E (2003) Preferences towards novel foodsin Cebu sapella: the role of nutrients and social influences. Physiol \& Behav 80(2):341349

Wang S, Lassoie J, Curtis P (2006) Farmer attitudes towards conservation in Jigme Singye Wangchuck National Park, Bhutan. Env Conserv 33(2): 148-156 
Webber A, Hill C, Reynolds V (2007) Assessing the failure of a community-based human wildlife conflict mitigation project in Budongo Forest Reserve, Uganda. Oryx 41(2): 177-184 Woodroffe R, Thirgood, S, Rabinowitz S (2005) The impact of human-wildlife conflict on natural systems. In: People and Wildlife: Conflict or Coexistence?, ed by Woodroffe R, Thirgood S, Rabinowitz A, Cambridge, UK, Cambridge University Press, pp 1-13 


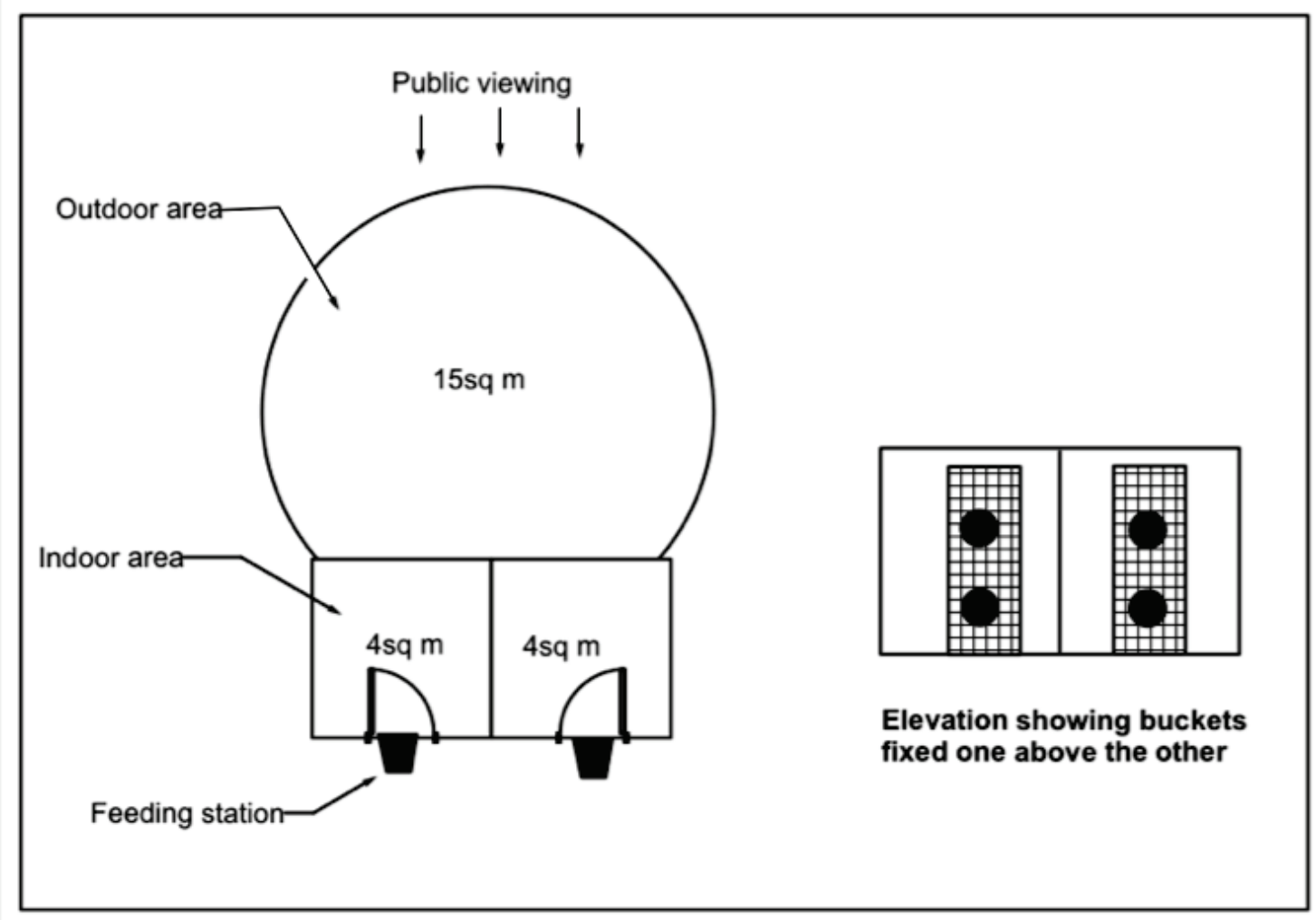

Figure 1: Diagram showing the position of the feeding stations in the enclosure 


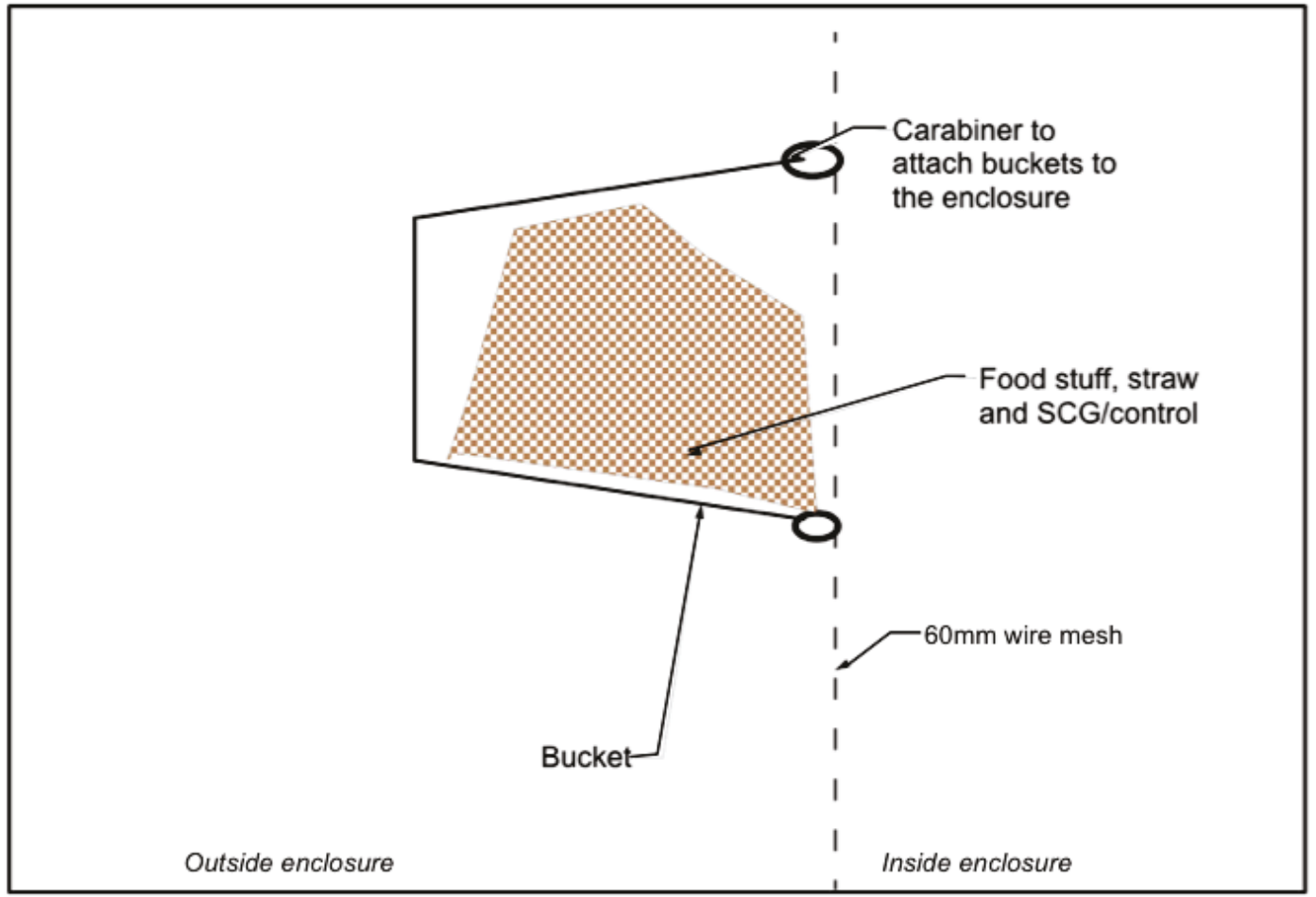

Figure 2: Diagram of the SCG feeding stations 


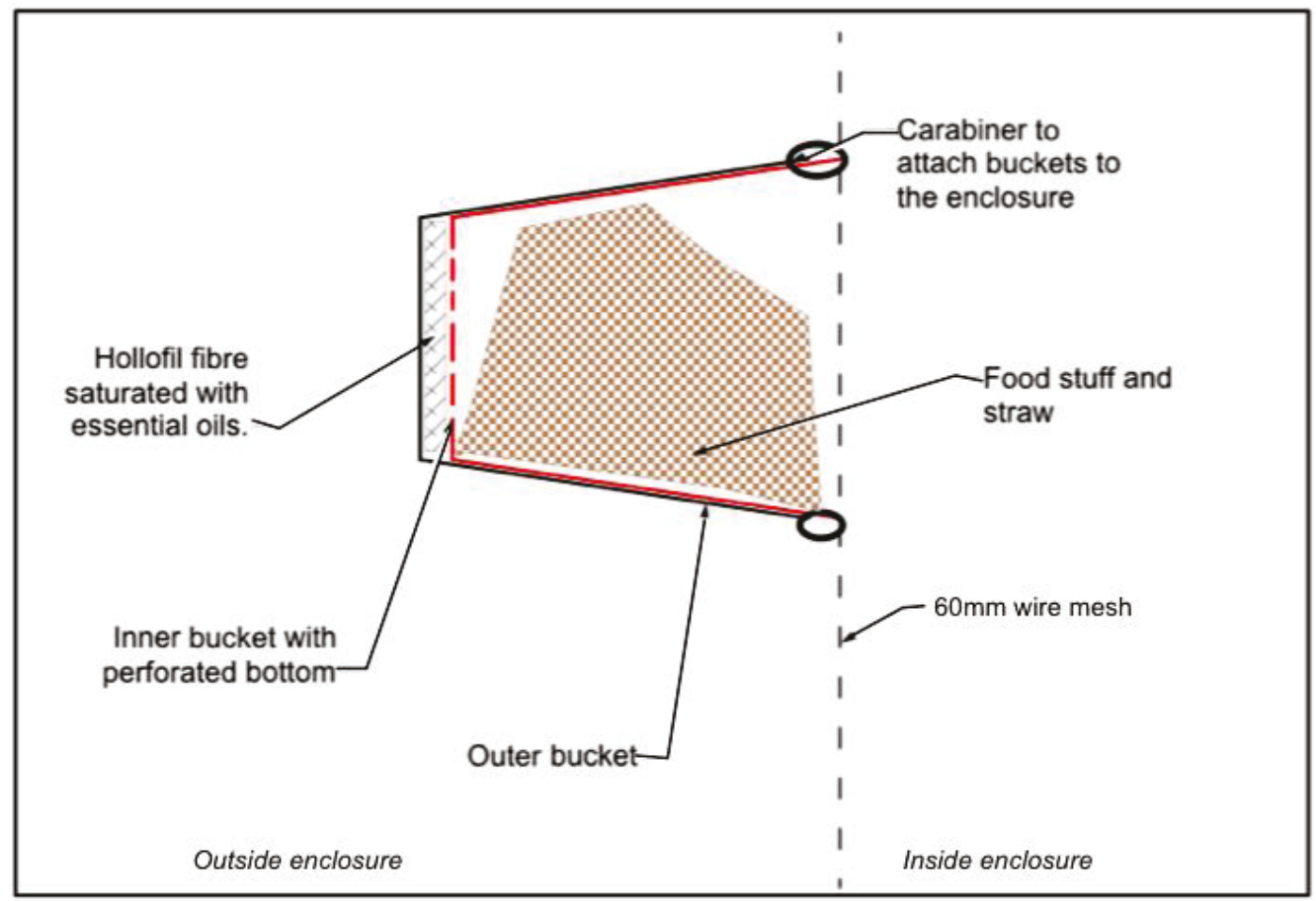

Figure 3: Diagram of the essential oils feeding station 


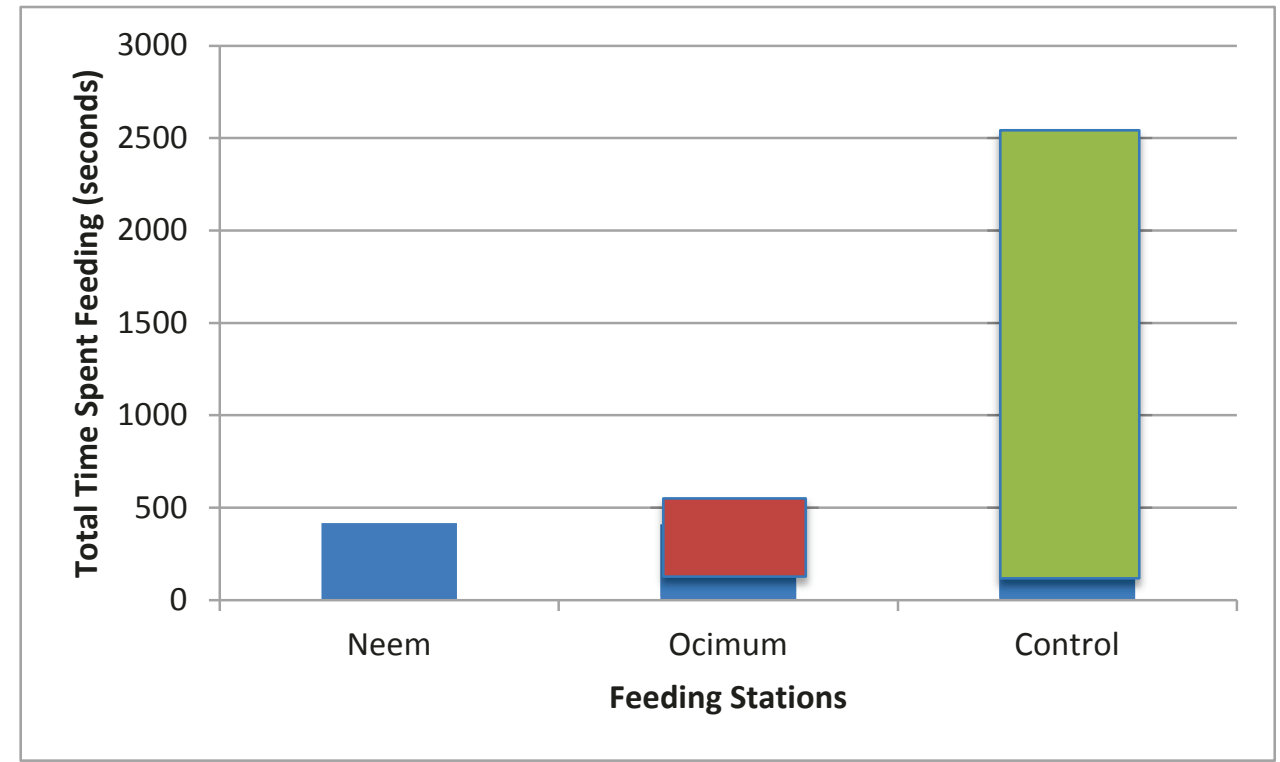

Figure 4: Durations of feeding at essential oils feeding stations compared to control feeding stations (ocimum and neem were tested separately against the control) 


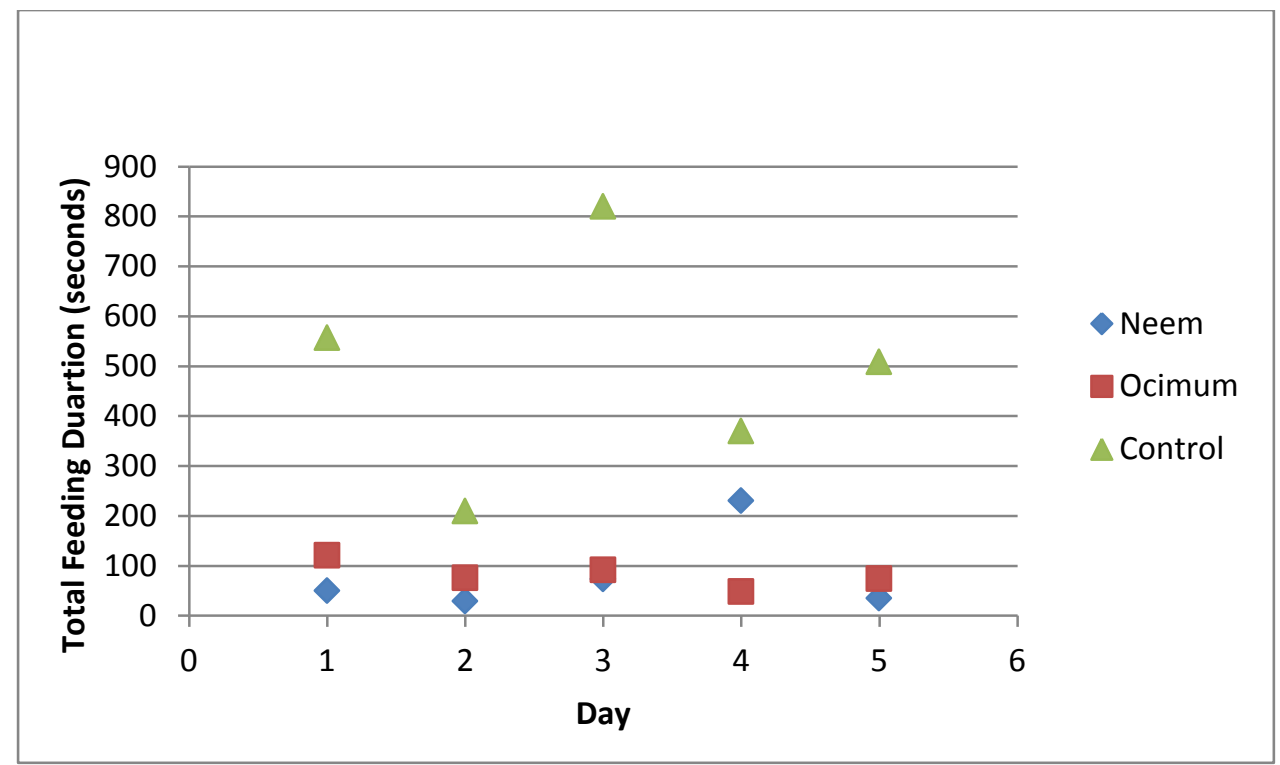

Figure 5: Macaque feeding duration over 5 days for the essential oils (essential oils were tested separately against the controls) 


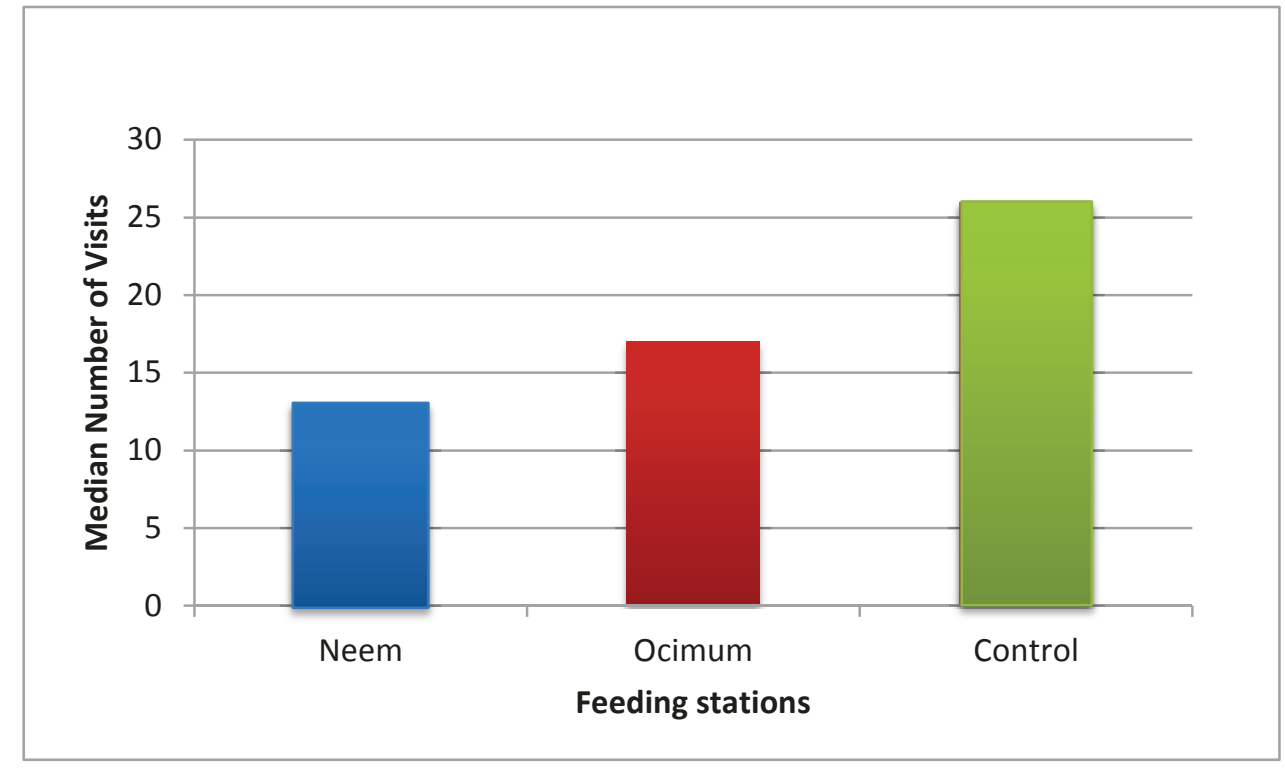

Figure 6: Frequency of feeding at essential oils feeding stations compared to control feeding stations 


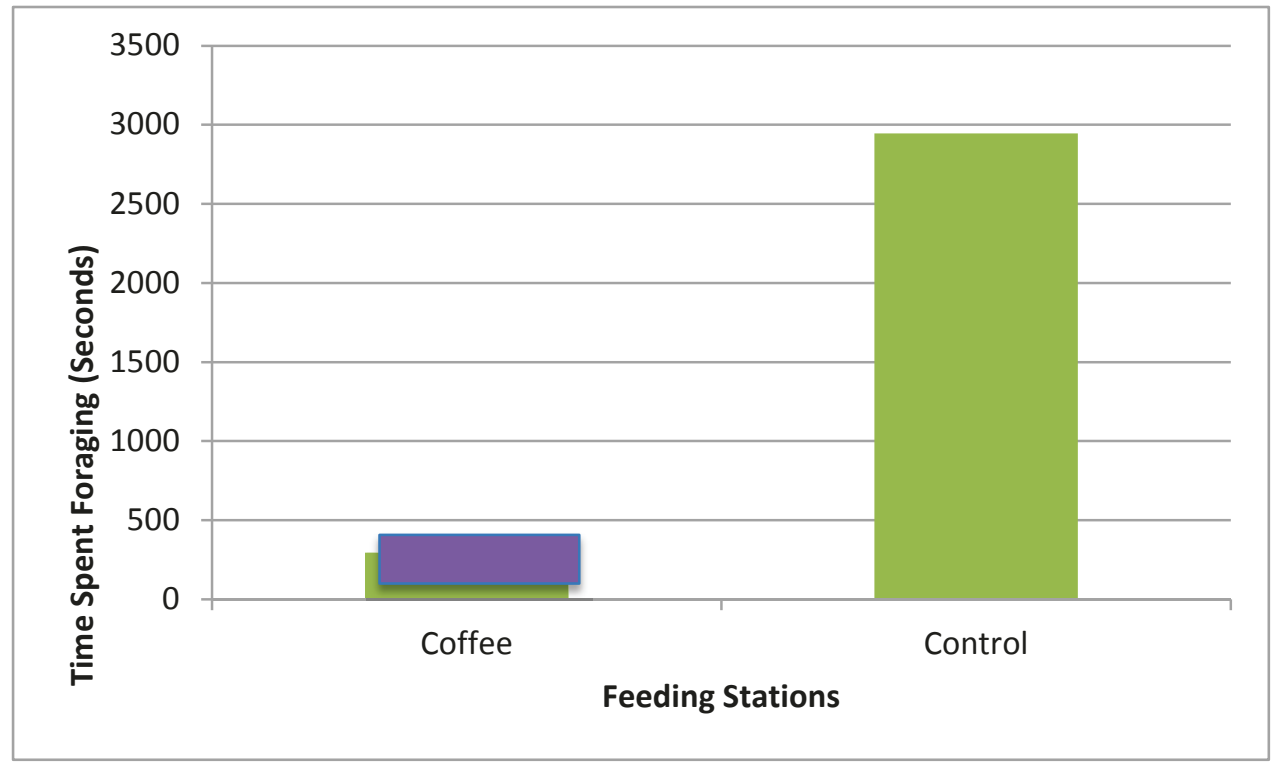

Figure 7: Total macaque feeding durations for the spent coffee grounds and control feeding stations 


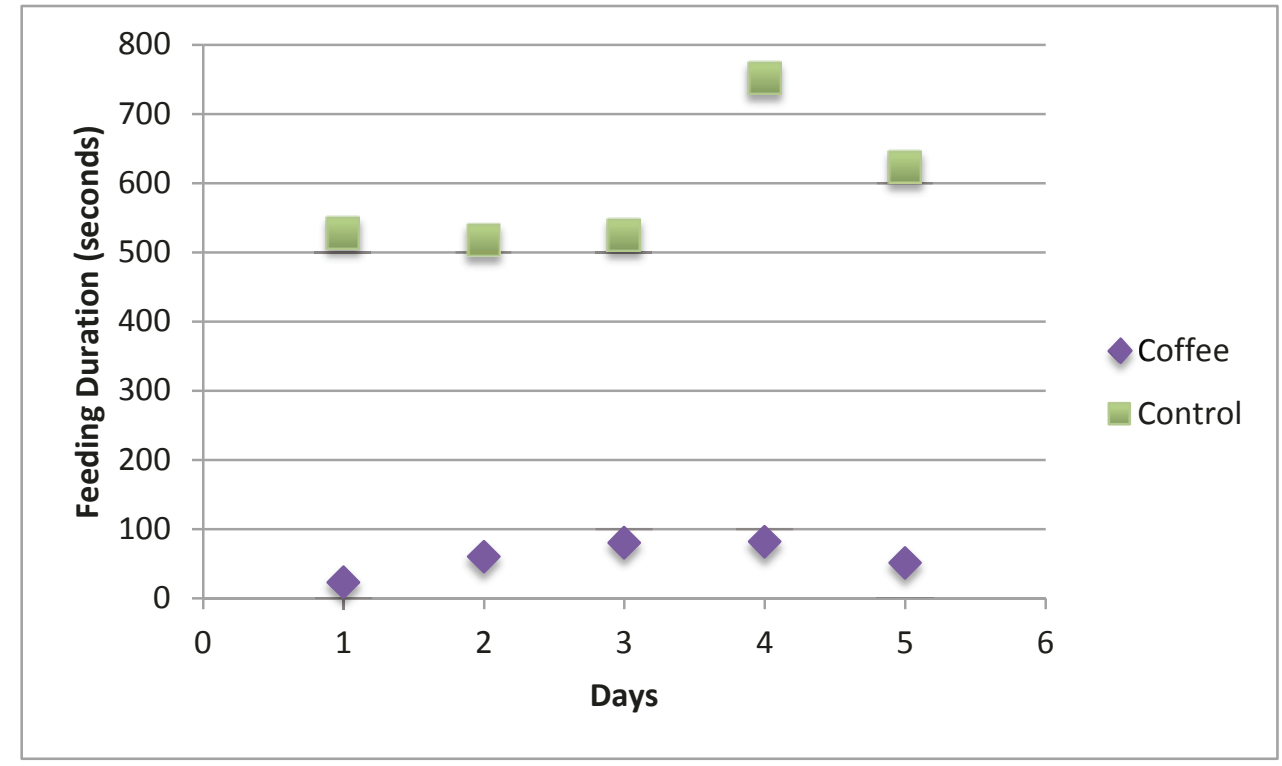

Figure 8: Macaque feeding durations over 5 days at the SCG/control feeding stations 


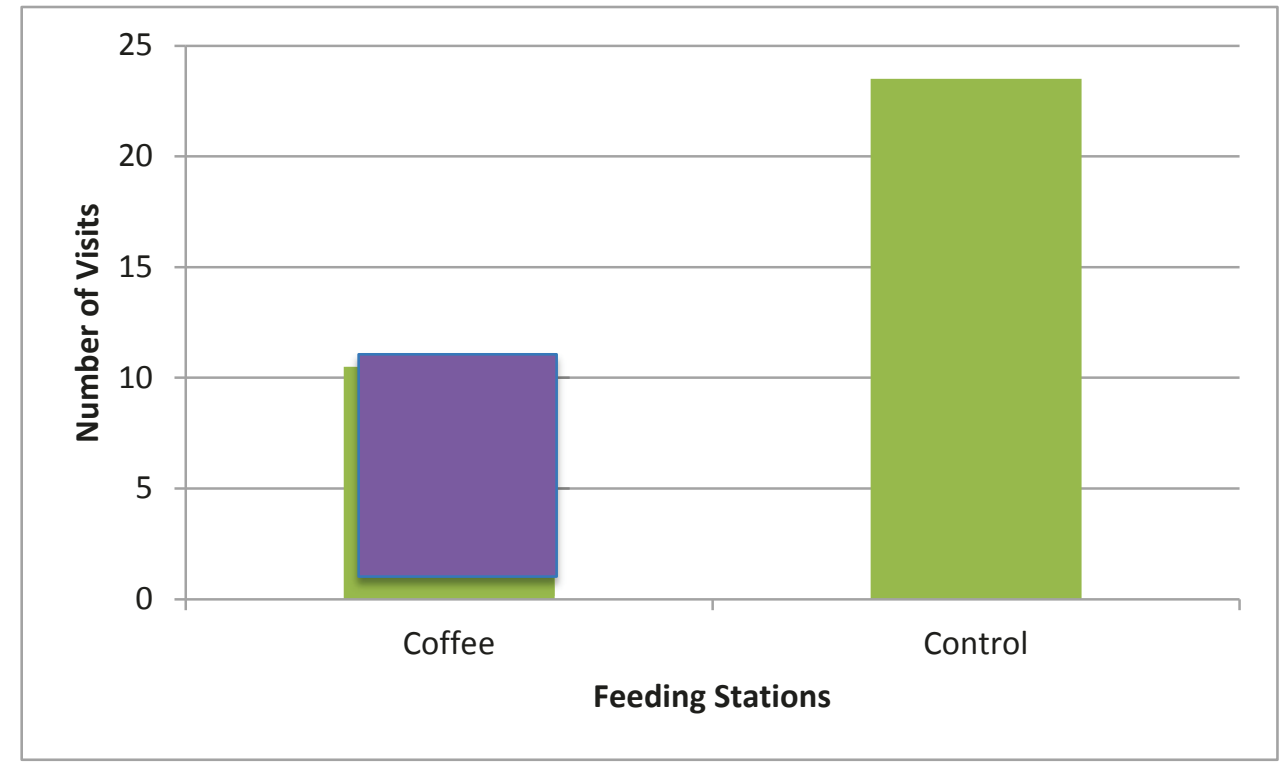

Figure 9: Total number of macaque visits to SCG/control feeding stations 